\title{
Redesigning the PheA Domain of Gramicidin Synthetase Leads to a New Understanding of the Enzyme's Mechanism and Selectivity ${ }^{\dagger}$
}

\author{
Brian W. Stevens, ${ }^{\ddagger}$ Ryan H. Lilien,,${ }^{\S, \#}$ Ivelin Georgiev, ${ }^{\S, \|}$ Bruce R. Donald,, , and Amy C. Anderson $*, \perp$ \\ Department of Biochemistry, Dartmouth Medical School, Hanover, New Hampshire 03755, Department of Computer Science, \\ Dartmouth College, Hanover, New Hampshire 03755, and Department of Pharmaceutical Sciences, University of Connecticut, \\ Storrs, Connecticut 06269
}

Received August 29, 2006; Revised Manuscript Received October 9, 2006

\begin{abstract}
The PheA domain of gramicidin synthetase A, a non-ribosomal peptide synthetase, selectively binds phenylalanine along with ATP and $\mathrm{Mg}^{2+}$ and catalyzes the formation of an aminoacyl adenylate. In this study, we have used a novel protein redesign algorithm, $K^{*}$, to predict mutations in PheA that should exhibit improved binding for tyrosine. Interestingly, the introduction of two predicted mutations to PheA did not significantly improve $K_{\mathrm{D}}$, as measured by equilibrium fluorescence quenching. However, the mutations improved the specificity of the enzyme for tyrosine (as measured by $k_{\mathrm{cat}} / K_{\mathrm{M}}$ ), primarily driven by a 56-fold improvement in $K_{\mathrm{M}}$, although the improvement did not make tyrosine the preferred substrate over phenylalanine. Using stopped-flow fluorometry, we examined binding of different amino acid substrates to the wild-type and mutant enzymes in the pre-steady state in order to understand the improvement in $K_{\mathrm{M}}$. Through these investigations, it became evident that substrate binding to the wildtype enzyme is more complex than previously described. These experiments show that the wild-type enzyme binds phenylalanine in a kinetically selective manner; no other amino acids tested appeared to bind the enzyme in the early time frame examined $(500 \mathrm{~ms})$. Furthermore, experiments with PheA, phenylalanine, and ATP reveal a two-step binding process, suggesting that the Phe A-ATP-phenylalanine complex may undergo a conformational change toward a catalytically relevant intermediate on the pathway to adenylation; experiments with PheA, phenylalanine, and other nucleotides exhibit only a one-step binding process. The improvement in $K_{\mathrm{M}}$ for the mutant enzyme toward tyrosine, as predicted by $K^{*}$, may indicate that redesigning the side-chain binding pocket allows the substrate backbone to adopt productive conformations for catalysis but that further improvements may be afforded by modeling an enzyme:ATP: substrate complex, which is capable of undergoing conformational change.
\end{abstract}

Several pharmacologically active compounds, including vancomycin, bacitracin, bleomycin, and cyclosporin, are produced by non-ribosomal peptide synthetases (NRPS). ${ }^{1}$ NRPS enzymes are composed of multiple modules that carry out distinct reactions using canonical and non-canonical

Funding Information: National Science Foundation (0133469 to A.C.A.), Research Corporation (RI0857 to A.C.A.), and National Institutes of Health (R01 GM65982 to B.R.D.).

* To whom correspondence should be addressed. Phone: (860)4866145. Fax: (860)486-6857. E-mail: Amy.Anderson@uconn.edu.

Department of Biochemistry.

$\S$ Department of Computer Science.

${ }^{\perp}$ Department of Pharmaceutical Sciences.

\# Current affiliation: Department of Computer Science and BantingBest Department of Medical Research, University of Toronto, Toronto, Ontario.

"Current affiliation: Department of Computer Science, Duke University, Durham, NC.

II Current affiliation: Department of Computer Science, Duke University, Durham, NC and Department of Biochemistry, Duke University Medical Center, Durham, NC.

${ }^{1}$ Abbreviations: NRPS, non-ribosomal peptide synthetase; A domain, adenylation domain; ATP, adenosine triphosphate; T, thiolation; $\mathrm{C}$, condensation; GrsA, gramicidin synthetase A; PheA, phenylalanine adenylation domain; AspA, aspartic acid adenylation domain; $\mathrm{PP}_{\mathrm{i}}$, inorganic pyrophosphate; NADH, $\beta$-nicotinamide adenine dinucleotide; AMP, adenosine monophosphate; AMPCPP, $\alpha, \beta$-methyleneadenosine 5'-triphosphate. amino acids as substrates and forming peptide or peptidelike products. The modular organization of the enzymes serves as a template for the chemical steps necessary to produce the peptide product. Adenylation (A) domains in NRPS enzymes bind the amino acid substrates and catalyze adenylation reactions using ATP and $\mathrm{Mg}^{2+}$ as cofactors. Previous studies $(1,2)$ have validated that the A domain plays the major role in ensuring specificity for amino acid activation. Several excellent reviews describe the modular structure and mechanism of the NRPS enzymes (3-6).

Gramicidin synthetase has served as a model NRPS system not only to understand fundamental mechanism but also as a template for redesign. Gramicidin synthetase A (GrsA), in concert with GrsB, produces a 5-residue monomer, the dimer of which forms the antibiotic, gramicidin. GrsA is composed of three domains, often called PheATE: a phenylalanineadenylating domain (PheA), a thiolation (T) domain, which is covalently bound in the holo state to a phosphopantetheine arm that attacks the aminoacyl adenylate to form an aminoacyl thioester, and an epimerization (E) domain, which epimerizes the L-phenylalanine aminoacyl thioester to a D-phenylalanine aminoacyl thioester.

If a means to edit or rearrange this template of enzyme modules was available, a new mechanism for producing 
novel derivatives of naturally occurring products could be used to produce molecules with potential novel pharmacological properties. Redesigning NRPS enzymes to synthesize new products-products that may have potential as therapeutic agents-could be accomplished by switching the monomer incorporated in the elongated polypeptide, modifying the polypeptide length or connectivity, or changing the covalent modifications made to the polypeptide $(5,7,8)$.

Previous efforts to discover the basis of selectivity of native NRPS domains include kinetic and thermodynamic analysis of the reactions in PheATE with Phe and alternate amino acid substrates $(9,10)$. Equilibrium fluorescence quenching experiments suggest that PheATE binds several amino acid substrates indiscriminately; seven of ten $K_{\mathrm{D}}$ values are within a factor of 2 , and all are within 1 order of magnitude. Steady-state kinetic data reveal that L- and D-Phe are preferred substrates. Amino acids such as L-Trp, L-Tyr, and L-Leu may still serve as substrates, but catalysis is 13-, 310-, and 16-fold less efficient, respectively. Pre-steady state, rapid-quench experiments in which an amino acid substrate, ATP, PheATE, and $\mathrm{Mg}^{2+}$ are mixed and then quenched at specific intervals show that adenylated L- and D-Phe-AMP are formed 10 times faster than other amino acyl adenylates.

Other studies (11) have shown that the apo-tyrocidine synthetase 1 A domain properly forms an amino acyl adenylate in the presence of the cognate substrate, Phe. However, a secondary catalytic activity, which causes $\mathrm{P}_{\mathrm{i}}$ release, is triggered in the presence of miscognate amino acids. The authors suggest a correlation between substrate specificity, suboptimal conformational states, and ATPase activity.

In order to redesign PheA to adenylate alternate substrates, site-directed mutagenesis has been used to replace active site residues identified as conserved in A domains that bind a common substrate and are presumed to interact with that substrate (these residues are called "signature sequences" for a specific substrate) (2). Mutation of two active site residues in PheA (T278M/A301G) alters the enzyme to selectively adenylate leucine. A one-point mutation to AspA (H322E) of a surfactin synthetase domain induces AspA to prefer asparagine over the natural substrate, aspartate. Creating and testing these mutant enzymes also highlighted one of the major difficulties in the redesign of A domains; every mutation resulted in an enzyme with lower catalytic efficiency than the wild-type enzyme. Even in the cases where substrate specificity is switched, the activity of the mutant enzyme, toward any substrate, was lower than the activity of the wild-type enzyme toward the natural substrate. These data suggest that alterations to the active site often perturb the enzyme from an optimal catalytic conformation.

Implementation of a novel protein redesign algorithm, $K^{*}$ (12), to redesign the PheA domain of GrsA to bind leucine identified two, 2-residue mutants, PheA(A301G/I330F) and PheA(A301G/I330W), which exhibit preferred binding for leucine over phenylalanine. Briefly, $K^{*}$ combines a statistical mechanics derived, ensemble-based approach to computing the binding constant with the speed and completeness of a branch-and-bound algorithm. It also features an efficient deterministic-approximation algorithm, capable of approximating a scoring function to arbitrary precision. Ensemble scoring, which uses a rotameric approximation to the partition functions of the bound and unbound states for PheA, is used to switch the enzyme specificity toward a given substrate by searching through a space of possible active site mutations. Ideally, $K^{*}$ would optimize transition state binding, that is, $K^{*}$ would search for a higher energy state presumed to be present at catalysis. However, in the absence of structural information for the transition state of these enzymes, $K^{*}$ is designed to maintain backbone contacts of the substrate in proximity to the nucleotide. In addition, the ensemble-based approach is designed to incorporate several conformations of the enzyme, some of which may approximate the transition state.

It is important to understand the specificity and selectivity of the NRPS enzymes in the natural system in order to manipulate that system to create novel products. Here, we present a new application of $K^{*}$ to redesign PheA to preferentially bind tyrosine, a larger amino acid than Phe instead of a smaller one (such as leucine) and with different electrostatic properties from those of Phe. The $K^{*}$ algorithm predicted several mutants of PheA to exhibit preferential binding to tyrosine. The introduction of two mutations to PheA improved the specificity of the enzyme $\left(k_{\text {cat }} / K_{\mathrm{M}}\right)$ for tyrosine in steady-state kinetic experiments, mostly by an improvement in $K_{\mathrm{M}}$, but the improvement was not enough to supplant phenylalanine as the preferred substrate. In order to understand the improvement in $K_{\mathrm{M}}$, we investigated the wild-type and mutant enzymes in pre-steady-state experiments using stopped-flow fluorometry. These experiments revealed new details regarding the selectivity and binding of substrate to the wild-type enzyme. PheA binds Phe in a kinetically selective manner, and the PheA-ATP-Phe complex displays a second binding mode, presumably a conformational change, which is nucleotide dependent. This new understanding of the wild-type enzyme, including the nucleotide dependence, can be applied to redesign more efficient mutant enzymes.

\section{EXPERIMENTAL PROCEDURES}

$K^{*}$ Application to Tyr Redesign. $K^{*}$ was applied as described previously (12), except the search was performed to find mutant sequences that score well with tyrosine in the active site. The crystal structure of PheA bound to AMP (13) was used to define the location of the nine active site residues (D235, A236, W239, T278, I299, A301, A322, I330, and C331) that were allowed to mutate (any two at a time). The structure of the PheA active site and location of these nine residues are shown in Figure 1. Residues D235, A236, A301, A322, I330, and C331 were allowed to mutate to any of 9 hydrophobic amino acids (GAVLIFYWM); residues W239, T278, and I299 were allowed to mutate to any of 14 hydrophobic or polar/uncharged amino acids (GAVLIFYWMSTHNQ). Sequences that passed the volume filter were scored by $K^{*}$.

Cloning and Mutagenesis. PheA was cloned from GrsA (PheATE) using primers described previously (14) that added a 5' NcoI restriction site (5'-ATATCCATGGTAAACAGTTCTAAAAG-3') and a $3^{\prime}$ BamHI site (5'-TCTCGGATCCTAATACATCCTGCCAG-3'); underlined bases are modified from GrsA sequence, and bold designates restriction sites. The PCR products and the expression vector pQE-60 (Qiagen) were digested with NcoI and BamHI, purified from a $1 \%$ agarose gel and extracted. The digested vector was 
a)

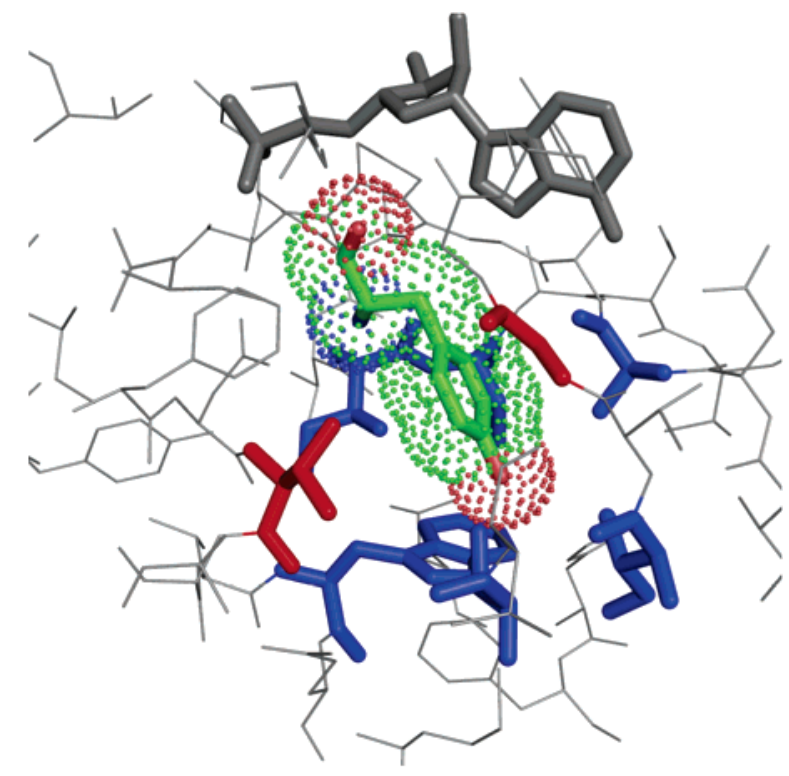

b)

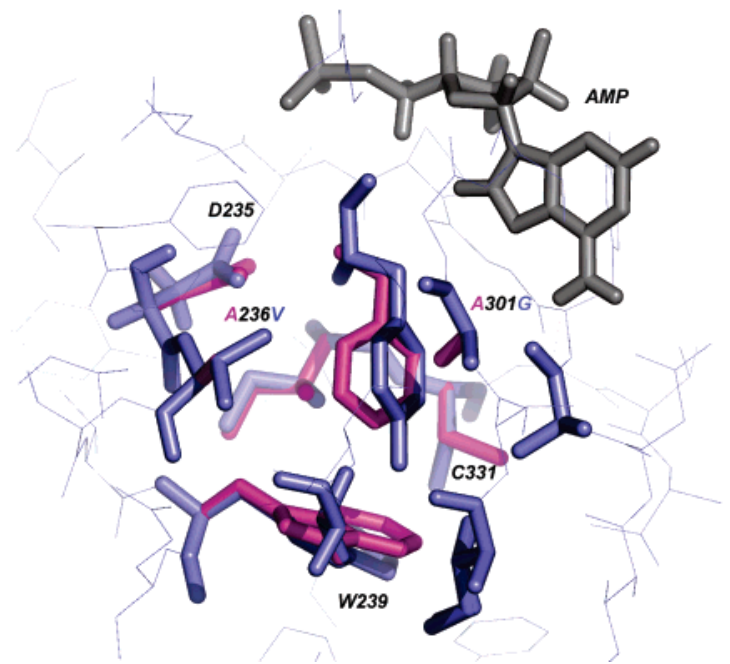

FIGURE 1: (a) Lowest-energy member of the ensemble for PheA(A236V/A301G). Shown are AMP (gray), tyrosine-CPK (with dots), 9 mutable residues (colored cylinders) $(236 \mathrm{~V}$ and $301 \mathrm{G}$ shown in red). (b) Superposition of the wild-type structure (magenta) with PheA(A236V/A301G) (blue). Residues showing conformational changes are shown in stick form.

treated with calf-intestine alkaline phosphatase, and the two fragments were ligated using T4 DNA ligase. M15 cells were transformed with the ligation reaction and streaked onto LB agarose plates containing ampicillin $(100 \mu \mathrm{g} / \mathrm{mL})$ and kanamycin $(25 \mu \mathrm{g} / \mathrm{mL})$. DNA was purified from clones using a QiaPrep mini-prep kit. Mutagenesis was performed using Strategene's QuickChange site-directed mutagenesis using the following primers: A236V (5'-GCCAGCATCTCTTTTGATGTATCTGTATGGGAGATGTTTATGGG3'), A236V-r (5'-CCCATAAACATCTCCCATACAGATACATCAAAAGAGATGCTGGC-3'), A301G (5'-CGTTAATTACAGGAGGCTCAGCTACCTCG-3'), and A301G-r ( $5^{\prime}$ CGAGGTAGCTGAGCCTCCTGTAATTAACG-3'). Construct and mutants were verified using the following: $\mathrm{pQE}-$ Type III/IV sequencing primer (5'-CGGATAACAATTTCACACAG-3'), pQE-promoter region sequencing primer (5'CCCGAAAAGTGCCACCTG-3'), pQE-reverse sequencing primer (5'-GTTCTGAGGTCATTACTGG-3'), Aseq (5'-
GTCAATTTGCCAGCATCTC-3'), and Arevseq (5'-CTTCTCTCCTGGAACAAACGGG-3').

Expression and Purification. A saturated culture of M15 cells, transformed with the wild-type (wt) or mutant PheA construct, was used to inoculate $1 \mathrm{~L}$ of $\mathrm{LB}$, which contained ampicillin $(100 \mu \mathrm{g} / \mathrm{mL})$ and kanamycin $(25 \mu \mathrm{g} / \mathrm{mL})$, by a 1:100 dilution. The culture was incubated $\left(250 \mathrm{rpm}, 37^{\circ} \mathrm{C}\right)$ until $\mathrm{OD}_{600}=1.0$, when IPTG was added to a final concentration of $1 \mathrm{mM}$. The cells were grown for an additional $6 \mathrm{~h}$, harvested by centrifugation, and frozen at -80 ${ }^{\circ} \mathrm{C}$. Cells were thawed and chemically lysed using BugBuster reagent (Novagen). The lysate was clarified by centrifugation at $60000 \mathrm{~g}$ and filtered by a $0.22 \mu \mathrm{m}$ syringe-driven filter. The clarified lysate was applied to a Ni-NTA agarose (Qiagen) column that had been pre-equilibrated with $50 \mathrm{mM}$ Tris $-\mathrm{HCl}$ (pH 7.4), $500 \mathrm{mM} \mathrm{NaCl}$. The column was washed with equilibration buffer and eluted with buffer containing $250 \mathrm{mM}$ imidazole using a linear gradient over 3 column volumes. Fractions containing pure PheA were exchanged into $20 \mathrm{mM}$ Tris $-\mathrm{HCl}$ using PD-10 gel-filtration columns (GE Healthcare) and pooled. The concentration was determined by $\mathrm{A}_{280}$ using a molar absorption coefficient of 67520 $\mathrm{M}^{-1} \mathrm{~cm}^{-1}$.

Equilibrium Tryptophan Fluorescence Quenching. The fluorescence of tryptophan was monitored on an ISS PC1 spectrofluorimeter using an excitation of $295 \mathrm{~nm}$ and monitoring emission at $328 \mathrm{~nm}$. The fluorescence of a PheA solution $(0.1 \mu \mathrm{M}$ in $10 \mathrm{mM}$ Tris $-\mathrm{HCl}, \mathrm{pH} 7.0)$ was measured using an average of 10 measurements. The amino acid substrate was titrated into the solution starting at $0.3 \mu \mathrm{M}$ and was added until the observed quenching reached saturation. Acrylamide quenching was performed in the same manner for acrylamide concentrations from $0.3 \mu \mathrm{M}$ to 200 $\mathrm{mM}$. Data were fit to the following equation to obtain $K_{\mathrm{D}}$ : $\Delta F_{\mathrm{obs}}=\Delta F_{\max } /\left(1+K_{\mathrm{D}} /[\mathrm{S}]\right)$.

Steady-State Pyrophosphate-Release Assay. A coupled, continuous, spectrophotometric assay for inorganic pyrophosphate $\left(\mathrm{PP}_{\mathrm{i}}\right)(15)$ was used to determine the rate at which $\mathrm{PP}_{\mathrm{i}}$ was released into solution. Reactions were performed in a Molecular Dynamics SPECTRA max plus ${ }^{384}$ microplate reader at $22{ }^{\circ} \mathrm{C}$. The reaction mixture contained: $167 \mathrm{mM}$ Tris- $\mathrm{HCl}$ (pH 7.0), $1 \mathrm{mM}$ uridine-diphosphate-glucose, 345 $\mu \mathrm{M}$ glucose-1,6-bisphosphate, $1 \mathrm{mM} \beta$-nicotinamide adenine dinucleotide, $10 \mathrm{mM} \mathrm{MgCl} 2,2.5 \mathrm{mM}$ adenosine 5'-triphosphate, $3 \mathrm{mM}$ dithiothreitol, $10 \mu \mathrm{M}$ ethylenediaminetetraacetic acid, $2 \mathrm{U} / \mathrm{mL}$ uridine- $5^{\prime}$-diphosphoglucose pyrophosphorylase, $4 \mathrm{U} / \mathrm{mL}$ phosphoglucomutase, $4 \mathrm{U} / \mathrm{mL}$ glucose-6phosphate dehydrogenase, and $0.01 \mu \mathrm{M}$ PheA wild type or mutant. The reaction was started with the addition of amino acid substrate after a 10 min incubation to allow the removal of any contaminating $\mathrm{PP}_{\mathrm{i}}$. Substrate concentrations from 1 to $2000 \mu \mathrm{M}$ were used. The absorbance at $340 \mathrm{~nm}\left(\epsilon_{340}\right.$ $\mathrm{NADH}=6317 \mathrm{M}^{-1} \mathrm{~cm}^{-1}$ ) was monitored at $1 \mathrm{~min}$ intervals for $6 \mathrm{~h}$, and the collected data were analyzed using SOFTmax PRO to determine the observed rates of reaction. At least 200 colinear points $\left(r^{2} \geq 0.95\right)$ were used to determine observed rates. Kinetic constants $k_{\text {cat }}$ and $K_{\mathrm{M}}$ were determined from nonlinear regression of data to the Michaelis-Menten equation. Observed rates were enzyme and substrate concentration-dependent, and at the lowest substrate concentration, enzyme concentration was at least 100 -fold lower. 


\begin{tabular}{ccc}
\hline Table 1: $K^{*}$ Ranking and Scores for the Tyrosine Redesign \\
\hline & $K^{*}$ rank & $K^{*}$ score $\left(\times 10^{15}\right)$ \\
\hline A236V A301G & 1 & 4.03 \\
W239T A278W & 2 & 3.72 \\
W239I A278W & 3 & 3.30 \\
W239Y A278W & 4 & 2.43 \\
PheA (wt) & 199 & 0.0416 \\
\hline
\end{tabular}

Stopped-Flow Tryptophan Fluorescence Quenching. Tryptophan quenching was monitored for either $0.5 \mathrm{~s}$ (ATP and AMP) or $0.1 \mathrm{~s}$ (AMP and AMPCPP) under stopped-flow conditions using a KinTek2004 instrument equipped with a photomultiplier tube (PMT) to monitor fluorescence. The sample was excited at $295 \mathrm{~nm}$, and emission was monitored using a $340 \mathrm{~nm}$ specific band-pass filter. A solution of 8 $\mu \mathrm{M}$ PheA in $20 \mathrm{mM}$ Tris $-\mathrm{HCl}(\mathrm{pH}$ 7.4) was combined 1:1 with a solution of phenylalanine $(1-2000 \mu \mathrm{M})$, resulting in $4 \mu \mathrm{M}$ PheA and $0.5-1000 \mu \mathrm{M}$ phenylalanine in the sample cell. When present, ATP, AMP, AMPCPP, and $\mathrm{MgCl}_{2}$ were used at a final concentration of $1 \mathrm{mM}$. Between 15 and 20 transients were collected for each substrate concentration and averaged. Averaged transients were fit to the equation $y=$ $A \mathrm{e}^{k x}+C$, where $k$ is the observed rate $\left(k_{\text {obs }}\right), y$ is fluorescence, and $x$ is time. Observed rates $\left(k_{\mathrm{obs}}\right)$ and substrate concentrations were then fit to either a linear $\left(k_{\mathrm{obs}}=k_{+1}[\mathrm{~S}]\right.$ $\left.+k_{-1}\right)$ or hyperbolic $\left(k_{\mathrm{obs}}=K_{\mathrm{D}}[\mathrm{S}] /\left(\mathrm{k}_{+2}+[\mathrm{S}]\right)\right.$ function. $K_{+1}$ and $k_{-1}$ are the microscopic rate constants for the association and dissociation of substrate, and $k_{+2}$ is the forward rate of the second binding mode.

Calculation of Free Energies. The free energy of the enzyme unbound to substrate was assigned as zero. Free energies of transition states were calculated as

$$
\Delta G=-R T\left[\ln \left(k_{\mathrm{obs}}\right)-\ln \frac{k_{\mathrm{B}}}{T h}\right]
$$

where $k_{\mathrm{B}}$ equals Boltzmann's constant, $h$ equals Planck's constant, and $T$ represents the temperature in Kelvin. The free energy of equilibrium is calculated as

$$
\Delta G=-R T \ln K_{\mathrm{eq}}=-R T \ln \frac{k_{+1}}{k_{-1}}
$$

\section{RESULTS}

$K^{*}$ Redesign for Tyrosine. A computational search using $K^{*}$ was performed to predict mutant sequences of PheA that would exhibit improved binding for tyrosine and that would remain well-packed and properly orient the substrate relative to the nucleotide cofactor. Using the crystal structure of PheA (13) bound to AMP and a protocol in which nine amino acids in the active site were allowed to mutate and two residues could mutate at the same time (nine-choose-two) to bind tyrosine, 1174 of 4071 possible sequences passed a simple volume filter and were scored by $K^{*}$. Of these scored sequences, the top four, along with the wild-type sequence, are shown with their $K^{*}$ scores (Table 1), which should be viewed as relative numbers, not as absolute values of binding constants. PheA(A236V/A301G) is the highest scoring sequence in silico and was therefore created by mutating the native PheA gene, followed by expression and purification of the mutant protein.
Table 2: Equilibrium Measurements of $K_{\mathrm{D}}$ Values

\begin{tabular}{lccc}
\hline & PheA & PheA (A236V/A301G) & $\begin{array}{c}K_{\mathrm{D}} \text { improvement } \\
\text { (relative to wild type) }\end{array}$ \\
\hline$K_{\mathrm{D}}$ Phe & $3.51 \pm 0.3$ & $1.84 \pm 0.14$ & 1.92 -fold \\
$K_{\mathrm{D}}$ Tyr & $1.93 \pm 0.16$ & $1.32 \pm 0.21$ & 1.47 -fold \\
\hline
\end{tabular}

Equilibrium Tryptophan Fluorescence Quenching. Equilibrium fluorescence quenching experiments were performed with PheA and PheA(A236V/A301G) in order to measure the dissociation constant of the enzyme-substrate complex, $K_{\mathrm{D}}$. First, tryptophan fluorescence quenching experiments with PheA in the presence of varying concentrations of acrylamide were performed to determine the number of tryptophan residues contributing to the quenching effect. These results (not shown) reveal that quenching is linear with respect to acrylamide concentration from $1 \mu \mathrm{M}$ to $200 \mathrm{mM}$, indicating that fluorescence quenching is consistent with that of a single tryptophan residue, presumed to be Trp 239 in the active site. Next, equilibrium tryptophan fluorescence quenching of PheA and PheA(A236V/A310G) was measured in the presence of varying concentrations of phenylalanine and tyrosine. These equilibrium experiments indicate that PheA binds tyrosine with slightly higher affinity than phenylalanine $\left[K_{\mathrm{D}}=1.93 \mu \mathrm{M}\right.$ (tyrosine) and $K_{\mathrm{D}}=3.51 \mu \mathrm{M}$ (phenylalanine)]. Surprisingly, PheA(A236V/A301G) does not bind tyrosine with significantly higher affinity than the wild-type enzyme $\left[K_{\mathrm{D}}=1.32 \mu \mathrm{M}\right.$ (mutant) and $K_{\mathrm{D}}=1.93$ $\mu \mathrm{M}$ (wild type)] (Table 2). The slightly increased affinity between PheA(A236V/A301G) and phenylalanine relative to PheA is a consequence of the fact that a negative design procedure, a subroutine that would specifically select sequences that bind an alternate substrate but not bind the original substrate, is not incorporated in $K^{*}$. Experiments with AMP, amino acid substrate, and the enzyme show no significant differences from experiments without AMP, and control experiments with only AMP and the enzyme show no quenching effects.

Steady-State Kinetic Analysis. In order to probe the effects of the mutations predicted by $K^{*}$ on substrate specificity as measured through catalysis, steady-state kinetics studies of PheA, ATP, $\mathrm{Mg}^{2+}$, and various amino acids were performed. Measuring the degree of amino acid-dependent ATP consumption using a coupled spectrophotometric assay (15) that detects the rate at which inorganic pyrophosphate is released into solution shows that, among the naturally occurring amino acids, PheA specifically adenylates phenylalanine (Table 3 ). The specificity constant $\left(k_{\mathrm{cat}} / K_{\mathrm{M}}=1.3 \times 10^{-1} \mu \mathrm{M}^{-1} \mathrm{~min}^{-1}\right)$ for phenylalanine is over 13 times higher than that of the next closest naturally occurring amino acid, leucine, and over 150 times higher than that of tyrosine $\left(7.8 \times 10^{-4} \mu \mathrm{M}^{-1}\right.$ $\mathrm{min}^{-1}$ ). The $k_{\text {cat }}$ value for Phe agrees with previously published results using the same assay $(9,10)$, and the $K_{\mathrm{M}}$ value agrees within 1 order of magnitude. Among the nonnatural amino acids, 2-thienyl-alanine (Figure 2) is the only substrate tested that is preferred over phenylalanine. However, two other non-natural amino acids, 2-furylalanine, and 4-bromophenylalanine, are better substrates than all other naturally occurring amino acids tested except for phenylalanine. Differentiation of the specificity constant $\left(k_{\text {cat }} / K_{\mathrm{M}}\right)$ for the wild-type enzyme among substrates is dominated by $K_{\mathrm{M}}$, which is dependent on the rates of association and dissociation of the enzyme substrate complex as well as the 
Table 3: Steady-State Kinetic Analysis of Wild-Type and Mutant PheA with Amino Acid Substrates

\begin{tabular}{|c|c|c|c|c|c|c|}
\hline PheA (wt) & $K_{\mathrm{M}}(\mu \mathrm{M})$ & & $k_{\text {cat }}\left(\min ^{-1}\right)$ & & $k_{\mathrm{cat}} / K_{\mathrm{M}}\left(\mu \mathrm{M}^{-1} \min ^{-1}\right)$ & \\
\hline 2-thienyl-Ala & $0.71 \pm 0.29$ & & $0.26 \pm 0.0052$ & & $(3.6 \pm 1.6) \times 10^{-1}$ & \\
\hline Phe & $0.6 \pm 0.2$ & & $0.08 \pm 0.002$ & & $(1.3 \pm 0.54) \times 10^{-1}$ & \\
\hline 2-furyl-Ala & $21 \pm 1.1$ & & $0.99 \pm 0.017$ & & $(4.6 \pm 0.32) \times 10^{-2}$ & \\
\hline 4-Br-Phe & $90 \pm 2.8$ & & $2.4 \pm 0.028$ & & $(2.6 \pm 0.11) \times 10^{-2}$ & \\
\hline Leu & $250 \pm 35$ & & $2.5 \pm 0.23$ & & $(9.8 \pm 2.3) \times 10^{-3}$ & \\
\hline Tyr & $9000 \pm 1000$ & & $7.0 \pm 0.7$ & & $(7.8 \pm 1.7) \times 10^{-4}$ & \\
\hline Val & $3700 \pm 1600$ & & $2.2 \pm 1.1$ & & $(5.9 \pm 0.56) \times 10^{-4}$ & \\
\hline 4-Nitro-Phe & $2900 \pm 190$ & & $1.7 \pm 0.059$ & & $(5.9 \pm 0.58) \times 10^{-4}$ & \\
\hline Ala & $1800 \pm 1100$ & & $0.36 \pm 0.19$ & & $(2 \pm 2) \times 10^{-4}$ & \\
\hline PheA (A301G) & $K_{\mathrm{M}}(\mu \mathrm{M})$ & & $k_{\text {cat }}\left(\min ^{-1}\right)$ & & $k_{\mathrm{cat}} / K_{\mathrm{M}}\left(\mu \mathrm{M}^{-1} \min ^{-1}\right)$ & \\
\hline Phe & $\leq 1^{a}$ & & $0.07 \pm 0.005$ & $\downarrow$ & $\geq 7.0 \times 10^{-2}$ & $\downarrow$ \\
\hline 2-thienyl-Ala & $3.4 \pm 1$ & $\uparrow$ & $0.13 \pm 0.002$ & $\downarrow$ & $(3.9 \pm 1.3) \times 10^{-2}$ & $\downarrow$ \\
\hline 4-Br-Phe & $25 \pm 2$ & $\downarrow$ & $0.88 \pm 0.012$ & $\downarrow$ & $(3.5 \pm 0.33) \times 10^{-2}$ & $\uparrow$ \\
\hline Leu & $15 \pm 2.4$ & $\downarrow$ & $0.54 \pm .013$ & $\downarrow$ & $(3.5 \pm 0.64) \times 10^{-2}$ & $\uparrow$ \\
\hline 2-furyl-Ala & $42 \pm 3.8$ & $\uparrow$ & $0.72 \pm 0.02$ & $\downarrow$ & $(1.7 \pm 0.21) \times 10^{-2}$ & $\downarrow$ \\
\hline Tyr & $640 \pm 24$ & $\downarrow$ & $2.5 \pm 0.08$ & $\downarrow$ & $(4.0 \pm 0.28) \times 10^{-3}$ & $\uparrow$ \\
\hline Ala & $19 \pm 2.9$ & $\downarrow$ & $0.04 \pm 0.002$ & $\downarrow$ & $(2.1 \pm 0.4) \times 10^{-3}$ & $\uparrow$ \\
\hline 4-nitro-Phe & $540 \pm 370$ & $\downarrow$ & $0.3 \pm 0.16$ & $\downarrow$ & $(5.6 \pm 6.8) \times 10^{-4}$ & $=$ \\
\hline Val & $3300 \pm 1300$ & $=$ & $0.6 \pm 0.23$ & $\downarrow$ & $(1.8 \pm 1.4) \times 10^{-4}$ & $\downarrow$ \\
\hline PheA (A236V) & $K_{\mathrm{M}}(\mu \mathrm{M})$ & & $k_{\text {cat }}\left(\min ^{-1}\right)$ & & $k_{\text {cat }} / K_{\mathrm{M}}\left(\mu \mathrm{M}^{-1} \min ^{-1}\right)$ & \\
\hline Phe & $\leq 1 *$ & & $0.05 \pm 0.001$ & $\downarrow$ & $\geq 5.0 \times 10^{-2}$ & $\downarrow$ \\
\hline 2-thienyl-Ala & $5.3 \pm 0.71$ & $\uparrow$ & $0.16 \pm 0.003$ & $\downarrow$ & $(3.1 \pm 0.46) \times 10^{-2}$ & $\downarrow$ \\
\hline 4-Br-Phe & $12 \pm 1.2$ & $\downarrow$ & $0.27 \pm 0.003$ & $\downarrow$ & $(2.2 \pm 0.24) \times 10^{-2}$ & $=$ \\
\hline 2-furyl-Ala & $14 \pm 0.9$ & $\downarrow$ & $0.16 \pm 0.002$ & $\uparrow$ & $(1.1 \pm 0.084) \times 10^{-2}$ & $\downarrow$ \\
\hline Leu & $80 \pm 3.1$ & $\downarrow$ & $0.47 \pm 0.01$ & $\downarrow$ & $(5.9 \pm 0.37) \times 10^{-3}$ & $\downarrow$ \\
\hline Tyr & $180 \pm 3.7$ & $\downarrow$ & $0.68 \pm 0.004$ & $\downarrow$ & $(3.8 \pm 0.1) \times 10^{-3}$ & $\uparrow$ \\
\hline 4-nitro-Phe & $260 \pm 7.9$ & $\downarrow$ & $0.33 \pm 0.02$ & $\downarrow$ & $(1.3 \pm 0.1) \times 10^{-3}$ & $\uparrow$ \\
\hline Val & $35 \pm 2.6$ & $\downarrow$ & $0.0064 \pm 0.0001$ & $\downarrow$ & $(1.8 \pm 1.8) \times 10^{-4}$ & $\downarrow$ \\
\hline Ala & $130 \pm 29$ & $\downarrow$ & $0.016 \pm 0.0014$ & $\downarrow$ & $(1.2 \pm 0.38) \times 10^{-4}$ & $\downarrow$ \\
\hline
\end{tabular}

\begin{tabular}{|c|c|c|c|c|c|c|}
\hline $\begin{array}{l}\text { PheA } \\
\text { (A236V/ } \\
\text { A301G) }\end{array}$ & $K_{\mathrm{M}}(\mu \mathrm{M})$ & & $k_{\text {cat }}\left(\min ^{-1}\right)$ & & $k_{\text {cat }} / K_{\mathrm{M}}\left(\mu \mathrm{M}^{-1} \min ^{-1}\right)$ & \\
\hline Phe & 1.46 & $\uparrow$ & 0.17 & $\downarrow$ & $1.2 \times 10^{-1}$ & $=$ \\
\hline 2-thienyl-Ala & $15 \pm 0.56$ & $\uparrow$ & $0.47 \pm 0.002$ & $\uparrow$ & $(3.1 \pm 0.1) \times 10^{-2}$ & $\downarrow$ \\
\hline 4-Br-Phe & $30 \pm 1.7$ & $\uparrow$ & $0.50 \pm 0.007$ & $\downarrow$ & $(1.7 \pm 0.1) \times 10^{-2}$ & $\downarrow$ \\
\hline 2-furyl-Ala & $32 \pm 0.5$ & $\uparrow$ & $0.47 \pm 0.001$ & $\downarrow$ & $(1.2 \pm 0.04) \times 10^{-2}$ & $\downarrow$ \\
\hline Leu & $61 \pm 3.6$ & $\downarrow$ & $0.63 \pm 0.013$ & $\downarrow$ & $(1.0 \pm 0.08) \times 10^{-2}$ & $\uparrow$ \\
\hline Tyr & $160 \pm 3.2$ & $\downarrow$ & $0.91 \pm 0.027$ & $\downarrow$ & $(5.8 \pm 0.3) \times 10^{-3}$ & $\uparrow$ \\
\hline 4-nitro-Phe & $180 \pm 10$ & $\downarrow$ & $0.67 \pm 0.005$ & $\downarrow$ & $(3.8 \pm 0.2) \times 10^{-3}$ & $\uparrow$ \\
\hline Ala & $7800 \pm 2900$ & $\uparrow$ & $0.66 \pm 0.23$ & $\uparrow$ & $(8.5 \pm 6.1) \times 10^{-5}$ & $\downarrow$ \\
\hline Val & $230 \pm 3.5$ & $\downarrow$ & $0.006 \pm 0.007$ & $\downarrow$ & $(2.8 \pm 3.2) \times 10^{-5}$ & $\downarrow$ \\
\hline
\end{tabular}

${ }^{a}$ The lowest concentration of Phe tested was $1 \mu \mathrm{M}$ because this concentration maintains an excess of substrate; therefore, $K_{\mathrm{M}}$ values for the single mutants toward Phe are $\leq 1 \mu \mathrm{M}$ because the rates of reaction for all concentrations of Phe were identical. Change relative to wild type is indicated for each reaction with the mutants: $\uparrow$ represents an increase, $\downarrow$ represents a decrease, and $=$ represents no statistical change.

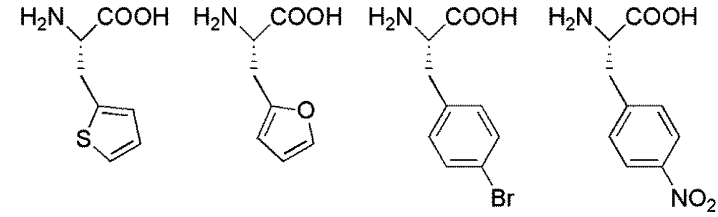

FIGURE 2: Non-natural amino acid analogues of phenylalanine. Left to right: 2-thienylalanine, 2-furylalanine, 4-bromophenylalanine, 4-nitrophenylalanine.

rate of conversion to product. The $K_{\mathrm{M}}$ values for the tested substrates span 4 orders of magnitude. Values for $k_{\text {cat }}$ are similar; besides phenylalanine and tyrosine, all the other amino acids tested have $k_{\text {cat }}$ values within 1 order of magnitude of each other. Phenylalanine has the lowest $k_{\text {cat }}$ value $\left(0.08 \mathrm{~min}^{-1}\right)$ among all those tested, and tyrosine has the highest $k_{\text {cat }}$ value $\left(7.0 \mathrm{~min}^{-1}\right)$; the low $k_{\text {cat }}$ value for phenylalanine agrees with previously published results $(9$, 10) and is a consequence of the fact that the assay is limited by the slow off-rates of the aminoacyl adenylate. Control experiments in the absence of amino acid substrate do not show any measurable changes over time.

The adenylation activity of $\mathrm{PheA}(\mathrm{A} 236 \mathrm{~V} / \mathrm{A} 301 \mathrm{G})$ is most specific for phenylalanine $\left(k_{\text {cat }} / K_{\mathrm{M}}=1.2 \times 10^{-1}\right)$, but specificity for tyrosine $\left(k_{\mathrm{cat}} / K_{\mathrm{M}}=5.8 \times 10^{-3}\right)$ is increased 7.4-fold compared to the wild-type PheA for tyrosine $\left(k_{\text {cat }} /\right.$ $\left.K_{\mathrm{M}}=7.8 \times 10^{-4}\right)$. The specificity of the double mutant also increased for 4-nitrophenylalanine (6.4-fold), it remained the same for leucine, and it decreased for all other substrates. Similar to PheA, values of $k_{\text {cat }}$ are within 1 order of magnitude for all but one substrate, valine. Specificity determination in the double mutant is still dominated by $K_{\mathrm{M}}$; values span 4 orders of magnitude.

The contributions of each single-point mutation (A236V and $\mathrm{A} 301 \mathrm{G}$ ) toward specificity for tyrosine appear to combine in the double mutant in a roughly additive manner. The specificity constant for each single mutant toward tyrosine increases from the PheA value of $7.8 \times 10^{-4} \mu \mathrm{M}^{-1}$ 


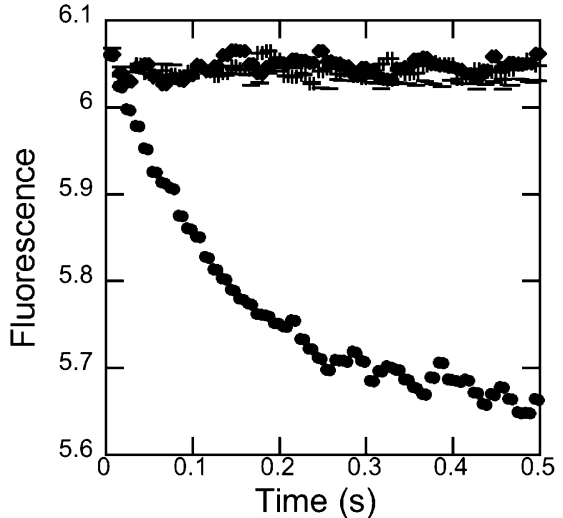

FIGURE 3: Quenching by phenylalanine requires ATP: - (buffer only), + (Phe), $\bullet$ (ATP), and (Phe and ATP).

$\min ^{-1}$ to values of $4.0 \times 10^{-3}$ and $3.8 \times 10^{-3}$ for PheA(A301G) and PheA(A236V), respectively. PheA(A236V/ $\mathrm{A} 301 \mathrm{G})$ has a specificity constant of $5.8 \times 10^{-3}$ toward tyrosine, slightly less than the sum of the single-point mutants. For each single mutant, the $k_{\text {cat }}$ decreased from the fastest observed rate of $7.0 \mathrm{~min}^{-1}$ observed with the wildtype enzyme to a value of $2.5 \mathrm{~min}^{-1}$ for PheA(A301G) and $0.68 \mathrm{~min}^{-1}$ for PheA(A236V). The $k_{\text {cat }}$ of PheA(A236V/ A301G), $0.91 \mathrm{~min}^{-1}$, was between that of the two single mutants. The $K_{\mathrm{M}}$ values of the single mutants for tyrosine are both significantly lower than that of the wild type, dropping from $9000 \mu \mathrm{M}$ in PheA to $640 \mu \mathrm{M}$ for PheA(A301G) and $180 \mu \mathrm{M}$ for $\mathrm{PheA}(\mathrm{A} 236 \mathrm{~V})$. The $K_{\mathrm{M}}$ value of the double mutant was lower than either of the single mutants, decreasing to $160 \mu \mathrm{M}$.

Pre-Steady-State Kinetic Analysis. The improvement in $K_{\mathrm{M}}$ for the double mutant toward tyrosine and the lack of proportional changes in $K_{\mathrm{D}}$ indicate a potentially incomplete picture of PheA selectivity. In order to gain a better understanding of PheA substrate binding and specifically to investigate whether the observed changes in $K_{\mathrm{M}}$ indicate a possible change in the rates of association and dissociation, fluorescence quenching experiments were performed under stopped-flow conditions to measure microscopic rates of binding and dissociation $\left(k_{+1}\right.$ and $\left.k_{-1}\right)$, as well as to determine dissociation constants.

Pre-equilibrium fluorescence quenching experiments show that the only detectable binding event in the first $500 \mathrm{~ms}$ is that between the wild-type PheA and its natural substrate, phenylalanine, in the presence of nucleotide and $\mathrm{Mg}^{2+}$ (Figure 3). Fluorescence decay curves measured for $500 \mathrm{~ms}$ for other amino acids such as tyrosine, valine, alanine, and leucine in the presence of PheA, ATP, and $\mathrm{Mg}^{2+}$ superimpose with curves from buffer alone. These results were expected because catalytic rates with valine and alanine as substrates are low and previous rapid quench experiments (9) showed that adenylated products for L-Leu and L-Tyr were observed only after $500 \mathrm{~ms}$. No quenching was detected within 500 ms using any substrate with the double mutant, with or without ATP and $\mathrm{Mg}^{2+}$.

When quenching is not observed it cannot be concluded that an interaction is not taking place between the enzymes and substrates. The association could occur at rates beyond the measurement capabilities of the instrument used (dead time of instrument $=1.4 \mathrm{~ms}$ ). The quenching could also be occurring so slowly that it is lost in the noise of the
Stop Flow Trp Quenching: PheA binding phe

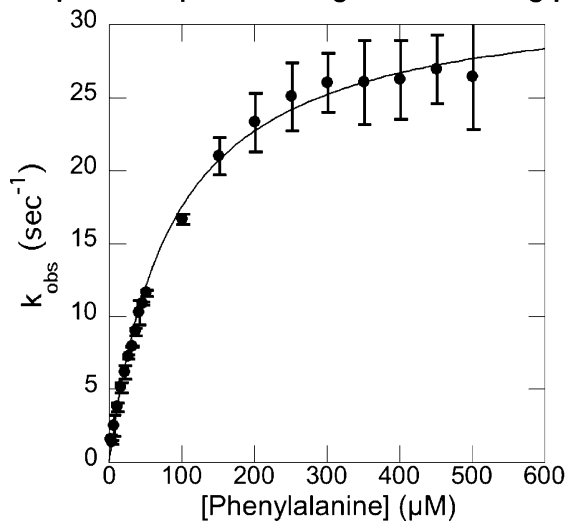

FIGURE 4: Observed rates of binding vs [Phe] are of the hyperbolic form-consistent with that seen in a two-step event. Error bars show 1 standard deviation of three measurements.

photobleaching, which occurs at a linear rate $\left(0.03 \mathrm{~s}^{-1}\right)$ much slower than the quenching event that occurs between PheA and phenylalanine in the presence of nucleotide, which occurs at an exponential rate ( $\mathrm{e}^{32 t}$ at the $K_{\mathrm{D}}$ of phenylalanine). In fact, the photobleaching rate limited the time course for these stopped-flow experiments to $500 \mathrm{~ms}$; binding events between the enzyme and leucine or tyrosine may occur, but we cannot observe them. A slow-binding event would be difficult to detect unless the amplitude of the signal was significantly larger than that of the natural substrate-enzyme interaction. However, under the conditions of the experiments and with the instrument used, a binding event between PheA and phenylalanine in the presence of nucleotide was the only one observed. This quenching event between PheA, phenylalanine, nucleotide, and $\mathrm{Mg}^{2+}$ reveals new details concerning the mechanism of the wild-type enzyme.

Quenching by phenylalanine in the presence of ATP and $\mathrm{Mg}^{2+}$ shows a hyperbolic dependence on phenylalanine (Figure 4), indicative of a two-step binding event (eq 1). The first step is characterized by the rapid establishment of an equilibrium between free substrate and an enzymesubstrate complex. The second step is usually a shift of the initial enzyme-substrate complex to a second complex characterized by a stronger interaction. This is the type of interaction seen in an induced-fit system of substrate recognition: an initial rapid collision event between the enzyme and substrate, followed by a slower conformational shift of the complex into an activated state.

$$
\begin{gathered}
\mathrm{E}+\mathrm{S} \underset{k_{-1}}{\stackrel{k_{+1}}{\longrightarrow}} \mathrm{ES} \stackrel{k_{2}}{\rightarrow} \mathrm{E}^{*} \mathrm{~S} \\
k_{\mathrm{obs}}=\frac{k_{2}[\mathrm{~S}]}{[\mathrm{S}]+K_{\mathrm{D}}}
\end{gathered}
$$

The data from the stopped-flow experiments can be used to determine several properties of the interaction. The relationship between the observed rate of binding and the concentration of substrate can be fit to eq 2 to determine the $K_{\mathrm{D}}$ of the initial binding event and the rate of the isomerization step, $k_{2}$. The initial near-linear portion of this hyperbolic curve ( $k_{\mathrm{obs}}$ vs [Phe]) can be fit to a line whose slope is an approximation of the second-order rate constant 
Table 4: Pre-Steady-State Kinetic Assay Results

\begin{tabular}{lcccc}
\hline \multicolumn{1}{c}{ complex } & $k_{+1}\left(\mu \mathrm{M}^{-1} \mathrm{sec}^{-1}\right)$ & $k_{-1}\left(\mathrm{~s}^{-1}\right)$ & $k_{2}\left(\mathrm{~s}^{-1}\right)$ & $K_{\mathrm{D}}(\mu \mathrm{M})$ \\
\hline PheA-ATP-Phe & 0.2 & 6.4 & 85 & 32 \\
PheA-AMP-Phe & 0.53 & 86.8 & & 164 \\
PheA-AMCPP-Phe & 0.44 & 54.9 & & 125 \\
\hline
\end{tabular}

of the initial collision event. For the PheA-phenylalanine interaction in the presence of ATP and $\mathrm{Mg}^{2+}$, the first 11 points can be fit to a line with an $r^{2}$ of 0.989 , giving a $k_{+1}$ of $0.21 \mu \mathrm{M}^{-1} \mathrm{sec}^{-1}$. The complete data set can be fit to a hyperbola with an $r^{2}$ of 0.996 that gives a $K_{\mathrm{D}}$ of $32 \mu \mathrm{M}$ and a $k_{2}$ of $85 \mathrm{~s}^{-1}$ (Table 4).

Quenching by phenylalanine in the presence of AMP and $\mathrm{Mg}^{2+}$ shows a linear dependence on phenylalanine (Figure $5)$, indicative of a one-step binding event. This type of interaction consists of a single, rapid collision event, with no shift to an activated complex (eq 3). The data for the PheA-phenylalanine interaction in the presence of AMP fit a line (eq 4) with an $r^{2}$ of 0.957 whose slope of $0.528 \mu \mathrm{M}^{-1}$ $\mathrm{sec}^{-1}$ is equal to the rate of association and whose $y$-intercept $\left(86.8 \mathrm{~s}^{-1}\right)$ is equal to the rate of dissociation. The quotient of the rates of association and dissociation gives a $K_{\mathrm{D}}$ for this interaction of $164 \mu \mathrm{M}$, signifying a weaker interaction than that between PheA-ATP-Phe (Table 4).

$$
\begin{gathered}
\mathrm{E}+\mathrm{S} \underset{k_{-1}}{\stackrel{k_{+1}}{\longrightarrow}} \mathrm{E}^{*} \mathrm{~S} \\
k_{\mathrm{obs}}=k_{+1}[\mathrm{~S}]+k_{-1}
\end{gathered}
$$

Quenching by phenylalanine in the presence of $\alpha, \beta$ methyleneadenosine 5 'triphosphate (AMPCPP), a nonhydrolyzable analogue of ATP, and $\mathrm{Mg}^{2+}$ shows a linear dependence on phenylalanine, similar to quenching seen in the presence of AMP (Figure 6). Fitting the data for AMPCPP in the same manner as described for AMP gives an $r^{2}$ value of 0.934 , a $k_{+1}$ of $0.44 \mu \mathrm{M}^{-1} \mathrm{sec}^{-1}$, a $k_{-1}$ of 54.9 $\mathrm{s}^{-1}$, and a $K_{\mathrm{D}}$ of $125 \mu \mathrm{M}$ (Table 4). Using a cutoff of 1 standard deviation, the data collected for PheA binding phenylalanine in the presence of AMP or AMPCPP are not significantly different at any point. In order to better resolve the fast-quenching events occurring in the presence of AMP and AMPCPP, stopped-flow data were collected over $0.1 \mathrm{~s}$, in contrast to the slower quenching seen in the presence of ATP collected over $0.5 \mathrm{~s}$. Data for PheA quenching by phenylalanine in the presence of AMP and AMPCPP appeared linear to the limit of data collection over $250 \mathrm{~s}^{-1}$. These high rates observed in the presence of AMP and AMPCPP are a sharp contrast to that collected in the presence of ATP whose fastest observed rate $\left(k_{\mathrm{obs}}\right)$ is below $30 \mathrm{~s}^{-1}$.

\section{DISCUSSION}

The predicted tyrosine-binding sequences predicted by $K^{*}$ seem reasonable on the basis of comparisons to known signature sequences and the predicted structures of the mutated enzymes. All of the mutations that scored in the top four appear in either a known signature sequence for tyrosine or other amino acids (2). A valine is present at position 236 in three signature sequences that bind ornithine, proline, or serine (the A domains are named Orn2, Pro, and

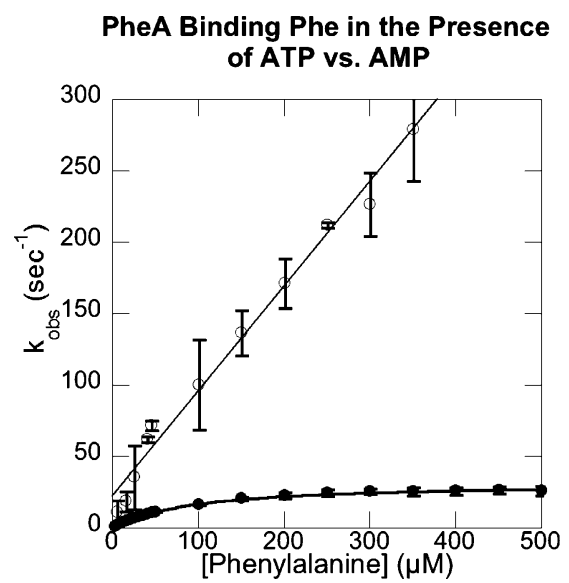

FIGURE 5: Observed rates of quenching by phenylalanine in the presence of AMP $(\mathrm{O})$ are linear with respect to [Phe], indicative of single-step binding. Observed rates in the presence of ATP $(\bullet)$ are much slower and have a hyperbolic relationship with [Phe]. Error bars show 1 standard deviation of three measurements.

Stop Flow Trp Quenching: PheA binding Phe

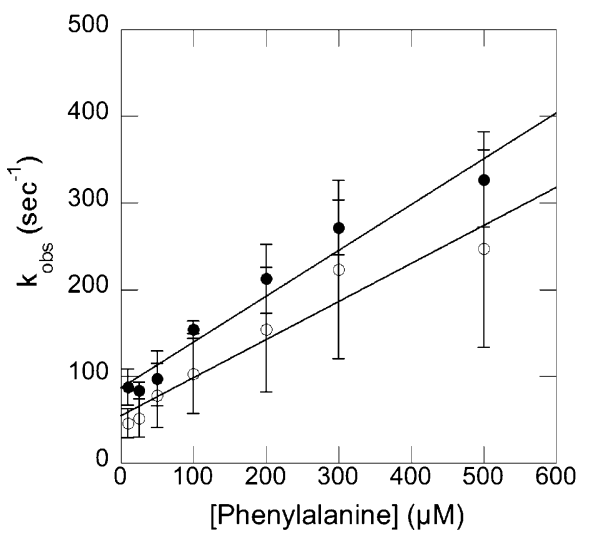

FIGURE 6: Observed rates of quenching in the presence of AMP (-) and AMPCPP $(O)$ have the same dependence on [Phe] and are not significantly different. Error bars show 1 standard deviation of three independent measurements.

Ser, respectively), and residue 301 is a glycine in most signature sequences, including one for tyrosine (Tyr2). Residue 239 is a threonine in one tyrosine signature sequence (Tyr1), and 239Y is found in a cysteine-binding sequence, Cys2. Position 278 is a tryptophan in a signature sequence that binds valine (Val1). Instances where $K^{*}$ suggests a mutation that exists in a signature sequence for the desired substrate show that $K^{*}$ has performed well scoring a sequence that evolution has already shown to be a successful mutation. In cases where a suggested mutation has not occurred for a given substrate, it is possible that evolution has not covered a given region of mutable space and some mutations that would improve specificity for a particular substrate have yet to arise through natural selection. Cases where a mutation exists for another substrate demonstrate that such mutations are at least allowable at that location.

The range of mutations explored by homology is inherently limited to those observed in nature; in contrast, $K^{*}$ explores an almost limitless set of possible mutations, thus providing an increased range of options in the redesign process. The signature sequence of PheA shares identity at 6 of 9 residues with one of the known tyrosine signature sequences, Tyr3 (2). The mutations that would be needed to make the PheA 
signature sequence identical with that of Tyr3 are W239S, I299V, and I330V. Two of these mutations were created; expression of PheA(I330V) was possible, but no soluble enzyme was detected in the extract of PheA(W239S), despite many variations in the conditions for expression. Therefore, although the mutations predicted by $K^{*}$ (A236V/A301G) have soluble expression levels below that of the wild-type enzyme, they do provide an option for testing a tyrosine redesign sequence.

Because a $K^{*}$ score represents an ensemble of conformations, with associated energies, for a given set of active site residues, there is no single conformation of enzyme and bound substrate that one can visualize to understand the complete interaction of substrate with a mutant. It is possible to visualize one or several of the low energy conformations that contribute to a $K^{*}$ score in order to understand the dominant interactions between enzyme and substrate. Inspection of several low energy conformers of PheA(A236V/ A301G) reveals how these two mutations might enhance the binding of tyrosine (Figure 1). Residues 236 and 301 are on opposite sides of the phenyl-binding pocket in the crystal structure (13). In the wild-type PheA both residues are alanines; mutating one to a larger valine, and the other to a smaller glycine, could allow the large aromatic ring to bind in the active site diagonally relative to the phenyl ring in the observed crystal structure. In fact, in the five lowest energy conformers of the redesigned Tyr enzyme, the single mutation, A236V, induces a slight, but important, conformational change that drops Trp $2391.4 \AA$ at the floor of the active site, allowing room for the para-hydroxyl group of tyrosine (Figure 1b).

The crystal structures of two other A domains have been described, both of which bind substituted aromatic rings: an A domain from DhbE, which binds $2^{\prime}, 3^{\prime}$-dihydroxybenzoate (16) and an adenylating domain from chlorobenzoate:CoA ligase (CBAL) that binds 4-chlorobenzoate (17). Interestingly, both of these enzymes contain one of the mutations predicted by $K^{*}$. DhbE contains a residue analogous to $301 \mathrm{G}$ and CBAL contains a residue analogous to $236 \mathrm{~V}$. All three species, PheA, DhbE and CBAL, have an aromatic residue at the floor of the substrate binding cavity that closes off the binding pocket. In the case of the redesigned tyrosine mutant for PheA, we allowed this residue to mutate to one which could potentially form a hydrogen bond with the hydroxyl group. However, $K^{*}$ maintained the aromatic character of this residue, but created a conformational change with the $236 \mathrm{~V}$ mutation to allow room for the para-hydroxyl.

Despite the fact that $K^{*}$ was not specifically designed to improve $K_{\mathrm{M}}$ values for a given substrate, steady-state pyrophosphate release assay data show improvement in PheA(A236V/A301G) specificity for tyrosine, driven by a change in $K_{\mathrm{M}}$. Examining the differences in $k_{\text {cat }} / K_{\mathrm{M}}$ for PheA and PheA(A236V/A301G) shows that values for all substrates decreased or stayed the same except for those for tyrosine and 4-nitro-phenylalanine, which increased over 7-fold for tyrosine and over 6-fold for 4-nitro-phenylalanine. The improvement for 4-nitro-phenylalanine is unexpected, but understandable given some limited structural similarities between tyrosine and 4-nitro-phenylalanine.

Differences in $K_{\mathrm{M}}$ appear to be the main determinant of the specificity of PheA. There is some variability among the $k_{\text {cat }}$ values, but it is orders of magnitude less than the variation seen for $K_{\mathrm{M}}$. Because the chemistry is occurring on the amino acid backbone, which is identical for all the substrates tested, it is reasonable to assume that once the enzyme-substrate complex attains the activated state required to proceed to catalysis, the catalytic process is similar for all substrates. A higher $K_{\mathrm{M}}$ indicates that the equilibrium between activated enzyme-substrate complex and free enzyme favors the free enzyme, corresponding to a lower concentration of activated enzyme-substrate complex available to undergo catalysis. The large differences in $K_{\mathrm{M}}$ and $k_{\text {cat }} / K_{\mathrm{M}}$ indicate that the ability of different substrates to reach the necessary activated enzyme-substrate complex is not the same and that substrate binding is partially rate-limiting.

Stopped-Flow Kinetics Measures an Important PheAPhenylalanine Interaction. Only the natural enzymesubstrate interaction between PheA and phenylalanine is detectable by stopped-flow fluorescence quenching in the first $500 \mathrm{~ms}$. Furthermore, a unique interaction between PheA and substrate occurs that requires the presence of ATP. Interestingly, Mobashery et al. (18) proposed a bi-bi substrate addition and product release mechanism for the adenylation domain of the aminoglycoside-modifying enzyme, ANT(3")-Ii/AAC(6')-IId, from Serratia marcescens. In this mechanism, ATP then substrate bind the enzyme and inorganic pyrophosphate, followed by the modified aminoglycoside, release as products. An implication of this type of mechanism, which may be similar to PheA, is that ATP binding may influence the affinity or rate of substrate binding.

These pre-steady-state data might seem to contradict that collected by equilibrium fluorescence quenching, which show an indiscriminate binding of substrate that does not require nucleotide, but this is not the case. Experiments done to measure quenching at equilibrium are examining thermodynamic control of substrate binding. Stopped-flow experiments measure kinetic control of substrate binding. The equilibrium results show that given enough time, even in the absence of cofactor (AMP or ATP and $\mathrm{Mg}^{2+}$ ), many amino acids are able to bind the active site of PheA and cause quenching. However, also clear from the pre-steadystate results is that in the presence of cofactors and on the earliest time scale (500 $\mathrm{ms}$ in this case), only phenylalanine is able to bind. Although the rapid quench experiments determined that the A domain does not hydrolyze a misactivated aminoacyl AMP, rapid quench experiments could not examine whether kinetic selection occurs during substrate binding. The pre-steady-state stopped-flow experiments described in this work show that PheA is kinetically selective for phenylalanine early in the catalytic cycle, at the time of substrate binding.

The data from the pre-steady-state experiments also correlate with the data from the steady-state pyrophosphaterelease assay, which show that other amino acids are suitable substrates for catalytic turnover. Because the turnover rates for all substrates are similar, there is most likely a similar catalytic mechanism and a similar catalytic conformation for all substrates; however, the time frame in which this is adopted is not the same. Taken together, the results from the equilibrium binding and steady- and pre-steady-state experiments point to a degree of kinetic selectivity for the PheA-ATP-Phe interaction. The PheA-ATP-Phe complex isomerizes to an activated enzyme-substrate complex; 


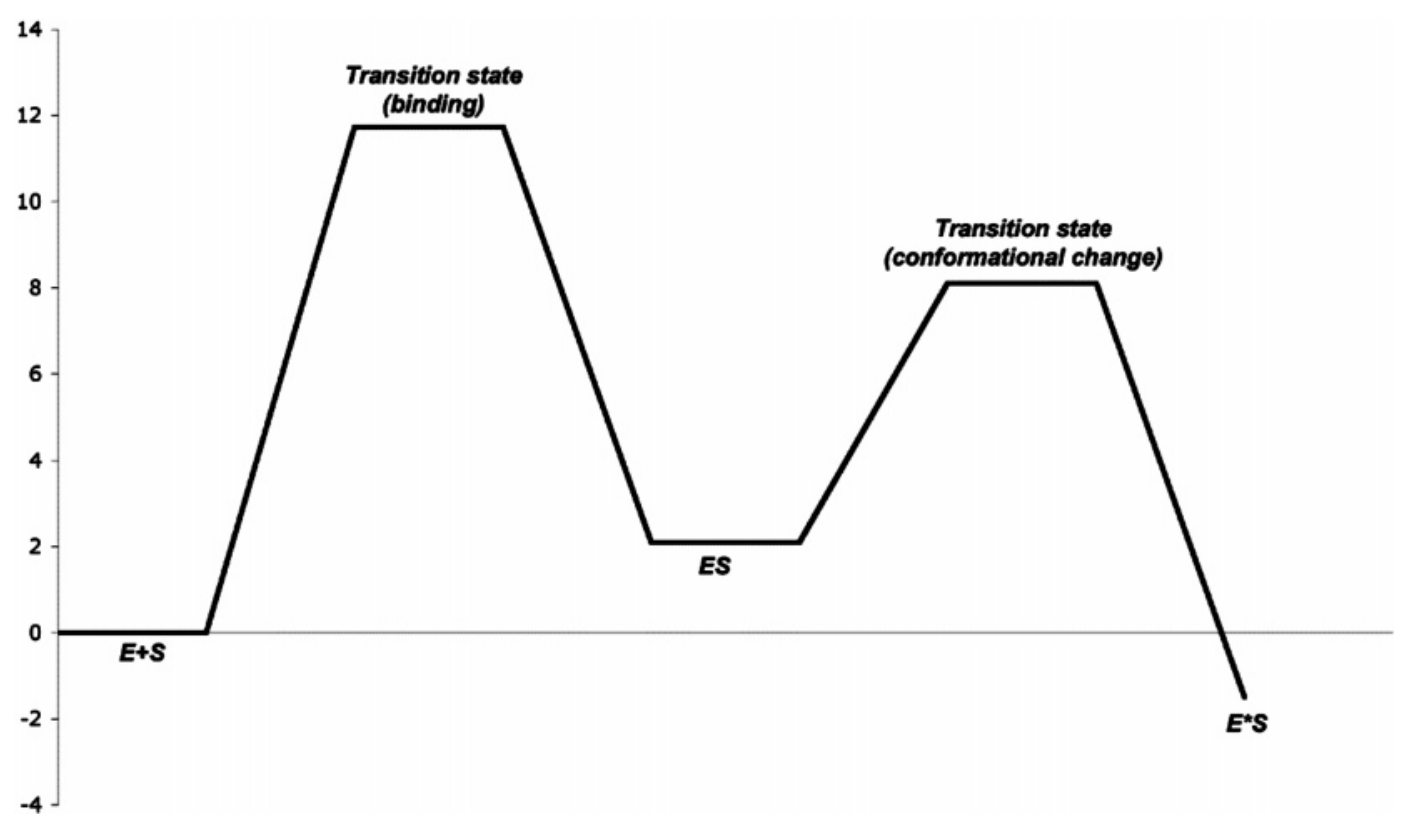

FIGURE 7: Free energy diagram representing the pre-steady-state steps for the wild-type enzyme binding phenylalanine.

the rate at which the enzyme adopts the activated conformation is much higher when bound to phenylalanine and lower when bound to other amino acids.

Using AMPCPP to study the PheA-phenylalanine interaction, we showed that, although it can serve as nucleotide necessary for quenching, it does not mimic ATP closely enough to achieve the two-step quenching event seen in the presence of ATP and $\mathrm{Mg}^{2+}$. The bond lengths between the phosphates and carbon are $1.81 \AA$ and between the phosphates and oxygen are $1.59 \AA$. The bond angle for $\mathrm{P}-\mathrm{C}-\mathrm{P}$ is $125.56^{\circ}$; the angle for $\mathrm{P}-\mathrm{O}-\mathrm{P}$ is $123.71^{\circ}$. These differences appear to be enough to disrupt the interaction between the $\beta / \gamma$-phosphates, coordinated $\mathrm{Mg}^{2+}$, enzyme, and substrate.

Steady- and pre-steady-state kinetics experiments are consistent with the existence of two phenylalanine-binding conformations of PheA. One of these conformations is an activated enzyme-substrate complex that is a catalytically relevant intermediate on the pathway toward the adenylation of phenylalanine (as in the case of PheA as it binds phenylalanine in the presence of ATP and $\mathrm{Mg}^{2+}$ ). The second conformation describes an enzyme-substrate complex that is likely to be representative of a product-releasing conformation (as in the case of PheA binding phenylalanine in the presence of AMP). PheA would normally be bound to an aminoacyl adenylate after catalysis, and it would need to release this product to prepare for another round of catalysis. The crystal structure of PheA with phenylalanine and AMP most likely represents the products of hydrolysis of the aminoacyl adenylate. The data support the following kinetic model of PheA binding phenylalanine in the presence of ATP:

$$
\begin{array}{r}
\text { PheA-ATP- } \mathrm{Mg}^{2+}+\text { Phe } \frac{\stackrel{0.2 \mu \mathrm{M}^{-1} \mathrm{~s}^{-1}}{\longrightarrow}}{6.4 \mathrm{~s}^{-1}} \text { PheA-ATP- } \\
\mathrm{Mg}^{2+}-\mathrm{Phe} \stackrel{85 \mathrm{~s}^{-1} \longrightarrow}{\longrightarrow} \mathrm{PheA}^{*}-\mathrm{ATP}-\mathrm{Mg}^{2+}-\mathrm{Phe}
\end{array}
$$

The ATP-bound conformation binds phenylalanine via a two-step process; the AMP-bound conformation binds phe- nylalanine via the following single-step process.

$$
\text { PheA-AMP- } \mathrm{Mg}^{2+}+\mathrm{Phe} \underset{86.8 \mathrm{~s}^{-1}}{\stackrel{0.53 \mu \mathrm{M}^{-1} \mathrm{~s}^{-1}}{\longrightarrow}} \mathrm{PheA}-\mathrm{AMP}-
$$

Note that the off-rate $\left(k_{-1}\right)$ is much higher in the presence of AMP over ATP, favoring the free PheA-AMP- $\mathrm{Mg}^{2+}$ and substrate.

Using the rate constants obtained in the pre-steady state, we calculated the free energy of the E+S and ES states as well as those for the transition states representing $\mathrm{E}+\mathrm{S}$ binding and the conformational change (Figure 7). From this graph, it is evident that substrate binding is rate-determining relative to the conformational change, for the wild-type enzyme binding phenylalanine. It is not clear, from these experiments, whether later catalytic steps are overall ratelimiting. Because binding events between other substrates and the wild-type enzyme and any substrate with the mutant enzyme cannot be measured in the early, $500 \mathrm{~ms}$ time frame, the transition state for these interactions will most likely involve a higher energy state.

The crystal structure of PheA (1AMU (13)) was solved with AMP resolved in the presumed nucleotide binding site. These new data show that phenylalanine interacts differently with PheA in the presence of ATP versus AMP and implies that the catalytically relevant substrate-binding conformation of PheA is not known at this time. It is likely that AMPand ATP-bound conformations of PheA are structurally very similar and that there are a few key interactions involving the terminal phosphates of ATP, $\mathrm{Mg}^{2+}$, and the backbone atoms of the bound amino acid substrate that are crucial for a catalytically competent conformation. Determining the nucleotide-sensing mechanism of PheA, and understanding how that mechanism relates to substrate binding, is necessary in order to improve the success of current NRPS modeling and redesign efforts.

There are three crystal structures of DhbE: an unliganded form, an adenylated form, and a hydrolyzed form with AMP 
and the aryl group bound independently (16); a comparison of these structures yields more information regarding the conformational changes around the bound ATP. The substrate in DhbE, 2', 3'-dihydroxybenzoate, binds in the same cavity as phenylalanine in PheA. The p-loop residues (core A3 or residues 190-200 in both DhbE and PheA) are at the entrance to the catalytic cavity, proximal to the nucleotide binding site, and are proposed to undergo a transition in the course of ATP and substrate binding, perhaps to isolate the catalytic cavity. In the three structures of DhbE, different residues in the p-loop exhibit ordered density in different structures, reflecting a conformational change dependent on changes in the states of substrate and nucleotide. The authors conclude that there is a clear conformational change in the residues interacting with ATP, before and after adenylation. Additionally, residue Arg428 in DhbE interacts with the $\alpha$-phosphate of ATP in the hydrolyzed complex but moves away in both the unliganded and the adenylated form.

In summary, interestingly, $K^{*}$ suggested mutations to PheA that slightly improved the binding constant, $K_{\mathrm{D}}$, but more importantly, generated a more catalytically competent conformation of the enzyme to bind tyrosine. This improvement in catalysis was most likely not a random event because only tyrosine and 4-nitrophenylalanine exhibited improved catalysis in the mutant enzyme relative to the wild-type enzyme. The structural changes induced by the A301G and A236V mutations appear to allow a para-hydroxyl group on phenylalanine to be more easily accommodated and thereby may also allow the backbone of tyrosine to adopt an orientation that can proceed to the transition state and eventually be in position to undergo catalysis. In contrast, phenylalanine may be bound in a more strained conformation in the mutated enzyme, and fewer Phe backbone orientations may be properly oriented to adopt the conformation of the transition state and therefore to be a proper substrate for catalysis, explaining the decrease in enzyme efficiency in the mutant. Finally, the pre-steady-state experiments suggest that PheA-ATP and phenylalanine initially collide and then undergo a second event, possibly a conformational change. Because $K^{*}$ selects sequences that represent a conformational ensemble and the ensemble itself embodies a degree of flexibility and a landscape of possible conformations, $K^{*}$ may select an ensemble that allows a productive conformational change to occur.

\section{ACKNOWLEDGMENT}

The authors thank Jared Cochran for assistance with stopped-flow fluorometry experiments, John MacMaster for discussions and assistance with preparing mutant PheA constructs, Jon Kull for use of the KinTek2004 instrument, and Harry Higgs for use of the ISS PC1 spectrofluorometer.

\section{REFERENCES}

1. Mootz, H. D., and Marahiel, M. A. (1997) The tyrocidine biosynthesis operon of Bacillus brevis: complete nucleotide sequence and biochemical characterization of functional internal adenylation domains, J. Bacteriol. 179, 6843-6850.

2. Stachelhaus, T., Mootz, H. D., and Marahiel, M. A. (1999) The specificity-conferring code of adenylation domains in nonribosomal peptide synthetases, Chem. Biol. 6, 493-505.

3. von Dohren, H., Keller, U., Vater, J., and Zocher, R. (1997) Multifunctional peptide synthetases, Chem. Rev. 97, 2675-2705.

4. Marahiel, M. A., Stachelhaus, T., and Mootz, H. D. (1997) Modular peptide synthetases involved in nonribosomal peptide synthesis, Chem. Rev. 97, 2651-2673.

5. Walsh, C. T. (2004) Polyketide and nonribosomal peptide antibiotics: Modularity and versatility, Science 303, 1805-1810.

6. Weber, T., and Marahiel, M. A. (2001) Exploring the domain structure of modular nonribosomal peptide synthetases, Structure 9, R3-R9.

7. Stevens, B. W., Joska, T. A., and Anderson, A. C. (2005) Progress toward re-engineering non-ribosomal peptide synthetase proteins: A potential new source of pharmacological agents, Drug Dev. Res. 66, 9-18.

8. Walsh, C. T., Chen, H. W., Keating, T. A., Hubbard, B. K., Losey, H. C., Luo, L. S., Marshall, C. G., Miller, D. A., and Patel, H. M. (2001) Tailoring enzymes that modify nonribosomal peptides during and after chain elongation on NRPS assembly lines, Curr. Opin. Chem. Biol. 5, 525-534.

9. Luo, L. S., Burkart, M. D., Stachelhaus, T., and Walsh, C. T. (2001) Substrate Recognition and Selection by the Initiation Module PheATE of Gramicidin S Synthetase, J. Am. Chem. Soc. 123, 11208-11218.

10. Luo, L. S., and Walsh, C. T. (2001) Kinetic Analysis of Three Activated Phenylalanyl Intermediates Generated by the Initiation Module PheATE of Gramicidin S Synthetase, Biochemistry 40, 5329-5337.

11. Pavela-Vrancic, M., Dieckmann, R., and von Dohren, H. (2004) ATPase activity of non-ribosomal peptide synthetases, Biochim. Biophys. Acta 1696, 83-91.

12. Lilien, R. H., Stevens, B. W., Anderson, A. C., and Donald, B. R. (2005) A novel ensemble-based scoring and search algorithm for protein redesign and its application to modify the substrate specificity of the gramicidin synthetase a phenylalanine adenylation enzyme, J. Comput. Biol. 12, 740-761.

13. Conti, E., Stachelhaus, T., Marahiel, M. A., and Brick, P. (1997) Structural basis for the activation of phenylalanine in the nonribosomal biosynthesis of gramicidin S, EMBO J. 16, 4174-4183.

14. Stachelhaus, T., and Marahiel, M. A. (1995) Modular structure of peptide synthetases revealed by dissection of the multifunctional enzyme GrsA, J. Biol. Chem. 270, 6163-6169.

15. Chen-Goodspeed, M., Vanhooke, J. L., Holden, H. M., and Raushel, F. M. (1999) Kinetic mechanism of kanamycin nucleotidyltransferase from Staphylococcus aureus, Bioorg. Chem. 27, $395-408$

16. May, J., Kessler, N., Marahiel, M., and Stubbs, M. (2002) Crystal structure of DhbE, an archetype for aryl acid activating domains of modular nonribosomal peptide synthetases, Proc. Natl. Acad. Sci. 99, 12120-12125.

17. Gulick, A., Lu, X., and Dunaway-Mariano, D. (2004) Crystal Structure of 4-Chlorobenzoate:CoA Ligase/Synthetase in the Unliganded and Aryl Substrate-Bound States, Biochemistry 43, $8670-8679$.

18. Kim, C., Hesek, D., Zajicek, J., Vakulenko, S., and Mobashery, S. (2006) Characterization of the Bifunctional AminoglycosideModifying Enzyme ANT(3")-Ii/AAC(6')-IId from Serratia marcescens, Biochemistry 45, 8368-8377.

BI061788M 